\title{
Digital Project and Showcase Evaluation Instrument
}

\section{Applicant Information}

\begin{tabular}{|c|c|c|}
\hline Applicant Name & Department & \\
\hline Rank/Role & $\begin{array}{l}\text { Agree to } \\
\text { recording? }\end{array}$ & $\begin{array}{l}\text { No, does not want to be recorded } \\
\quad \text { Yes, record \& publish presentation }\end{array}$ \\
\hline $\begin{array}{l}\text { Additional } \\
\text { Presenters }\end{array}$ & $\begin{array}{l}\text { Time } \\
\text { Selection }\end{array}$ & \multirow{2}{*}{$\begin{array}{ll}\square & \text { Lightning (1 min) } \\
\square & \text { Snapshot (3-5 min) } \\
\square & \text { Brief (8-10 min) }\end{array}$} \\
\hline $\begin{array}{l}\text { Project URL } \\
\text { (if any) }\end{array}$ & & \\
\hline Project Title & & \\
\hline
\end{tabular}

\section{Ratings}

$$
1=\text { Poor }
$$$$
2 \text { = Fair }
$$

$3=$ Satisfactory

$4=\mathrm{Good}$

$5=$ Excellent

Project / Topic Quality

Comments

Quality of Tools I Methods I Resources Used

$\square$

口

口

口

口

Comments

Appropriateness for Audience

Comments

Overall Rating (add up the rating numbers above)

If a tie breaker is needed, consider adding a Wow Factor rating (ie: how excited are you to see this presentation? 1-5) or consider the diversity of roles and departments represented among the applications. You can also extend the Showcase time frame by hours or days to include more participants.

\section{Open Evaluation}

CONCERNS: (including technical infrastructure required, time, copyright, topic, other)

\section{STRENGTHS:}

\section{Recommended Adjustments (if any)}

(include any adjustments that are fitting, such as changing presentation times to 1 minute, 2 minute, 3, minute, etc or combining presenters)

\section{Reviewer Name:}

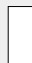

Reviewer Name:
Date: $\square$

$\square$ 